\title{
Andreï Tarkovski, Alexandre Sokourov, Otar Iosseliani (vus par Chris Marker, Anne Imbert, Julie Bertucelli) ou les guetteurs mélancoliques
}

\section{Didier Coureau}

\section{(2) OpenEdition}

\section{Journals}

Édition électronique

URL : http://journals.openedition.org/recherchestravaux/521

DOI : 10.4000/recherchestravaux.521

ISSN : 1969-6434

Éditeur

UGA Éditions/Université Grenoble Alpes

Édition imprimée

Date de publication : 15 mai 2012

Pagination : 103-118

ISBN : 978-2-84310-227-1

ISSN : 0151-1874

\section{Référence électronique}

Didier Coureau, « Andreï Tarkovski, Alexandre Sokourov, Otar losseliani (vus par Chris Marker, Anne Imbert, Julie Bertucelli) ou les guetteurs mélancoliques », Recherches \& Travaux [En ligne], 80 | 2012 mis en ligne le 15 novembre 2013, consulté le 08 septembre 2020. URL : http:// journals.openedition.org/recherchestravaux/521; DOI : https://doi.org/10.4000/recherchestravaux 521 


\section{Andreï Tarkovski, Alexandre Sokourov, Otar Iosseliani (vus par Chris Marker, Anne Imbert, Julie Bertucelli)ou les guetteurs mélancoliques}

En avril 20ıo, des personnalités du cinéma russe, parmi lesquelles figuraient Alexeï Guerman, Alexandre Sokourov, Youri Norstein (réalisateurs), Naoum Kleiman (directeur du Musée du cinéma de Moscou), et Andreï Palakhov (critique) cosignèrent une tribune intitulée "On ne l'aime pas!», attaquant Nikita Mikhalkov, à la tête de la puissante Union des cinéastes russes depuis une dizaine d'années. Opposition frontale à la mainmise d'un cinéaste officiel, qui fut tour à tour proche de Boris Eltsine, Vladimir Poutine, Dmitri Medvedev. Les propos sont virulents :

On n'aime pas la recherche obsédée de l'ennemi intérieur et la mise à pied des insoumis. Et surtout, on n'aime pas le fait que la discussion libre, la diversité des opinions, l'esprit de la liberté et de la démocratie aient quitté les murs de notre union depuis longtemps, pour céder la place à la mentalité unitariste, au faux patriotisme et à la servilité qui y sont imposés.

Si fin d'empire il y a eu, certains atavismes hérités du communisme sont réapparus depuis : nostalgie du totalitarisme et contrôle des institutions.

Nous sommes persuadés [poursuivent les signataires] que l'installation d'une telle ambiance dans l'union artistique, présidée par une personne "qui a beaucoup de pouvoir" risque d'accroître des tendances anticonstitutionnelles, antidémocratiques et immorales présentes dans notre société. On ne veut pas

I. Dans G. Lefort, «On ne l'aime pas!», <http://www.liberation.fr/culture/oror630977nikita-mikhalkov-on-ne-l-aime-pas> [mis en ligne le I9 avril 20I0]. 
participer à ce processus dangereux. Voilà pourquoi on a pris cette décision qui est pénible pour nous tous : sortir de l'Union des cinéastes².

La révolte peut aujourd'hui s'exprimer, l'information être diffusée en Russie et dans le monde entier, mais ce profond malaise n'en souligne pas moins une forme de réactualisation inquiétante de la surveillance de la création par le pouvoir. Près de vingt ans après la chute de l'Empire soviétique une autre fin, celle de l'Union des cinéastes, serait-elle programmée?

Dix ans auparavant, en avril 2000, le cinéaste français Leos Carax (lié alors avec le cinéaste lituanien Sharunas Bartas) adressait une réponse, dans une tribune de Libération, à une lettre parue dans la presse, cosignée entre autres par Nikita Mikhalkov, Alexeï Guerman et Alexandre Sokourov, alors alliés. S'adressant à deux d'entre eux, il écrivait :

Messieurs Alexandre Sokourov et Alexeï Guerman, vous êtes deux cinéastes importants, et vous êtes russes. Voilà deux vérités. Ce sont elles qui vous ont valu de signer avec quelques autres illustres de vos compatriotes une «lettre aux médias russes et étrangers", lettre mensongère, hypocrite et lâche, dont on a pu lire la traduction dans la presse française 3 .

Aux auteurs de ce pamphlet, qui mettait la Russie en position de victime face aux terroristes tchétchènes (recette bien connue), tout en attaquant les critiques occidentales, Leos Carax rétorquait :

Messieurs, vivez-vous au royaume de Machiavel? Ou dans un monde à l'envers? Car ce qui aurait dû vous rendre « désolés surtout", c'est la lâcheté de la France, de l'Europe et du monde face aux agissements de Eltsine hier, de son fiston Poutine aujourd'hui. Vous auriez dû vous élever depuis longtemps pour dénoncer notre bienveillance envers la folie mafieuse du premier, nos silences devant la barbarie du second ${ }^{4}$.

Et, de manière plus forte encore, il concluait :

En imitant les grimaces obscènes de votre nouveau héros (fausse paranoïa, fausse fierté blessée, vrai cynisme vieux comme le monde), vous ne vous conduisez ni en artistes ni en résistants comme vous faites semblant de le croire, mais en pantins nationalistes et négationnistes. Et vous condamnez votre si beau pays à sombrer plus et plus .

Un troisième texte, signé par l'historienne du cinéma Marilyne Fellous, dans le cadre d'une rétrospective du cinéma russe au Festival de Fribourg, en mars 2010 - juste avant la rupture au sein de l'Union des cinéastes -, donnait

2. Ibid.

3. L. Carax, «Lettre à deux cinéastes», publiée sur <http://www.liberation.fr/tribune/oror33I454nikita-mikhalkov-alexei-guerman-et-alexandre-sokourov-defendaient-l-intervention-en-tchetchenie-le-cineastefrancais-condamne-ce-geste-lettre-a-deux-cineastes-russes...> [mise en ligne le 20 avril 2000].

4. Ibid.

5. Ibid. 
à lire une synthèse intitulée : "Le cinéma russe : vingt-cinq ans déjà ${ }^{6}$ ", qui montrait le chemin parcouru depuis la présidence de Mikhaïl Gorbatchev, et le déclenchement conjoint de la perestroïka (restructuration) et de la glasnost (transparence) en 1985, jusqu'à celle de Dmitri Medvedev, en passant par Boris Eltsine (au moment de l'effondrement de l'Empire soviétique) et Vladimir Poutine (dirigeant officiel ou officieux de la Russie depuis 1999). Marilyne Fellous souligne que la libéralisation du cinéma eut lieu dès I986, avec l'«acte d'indépendance" du cinéma soviétique vis-à-vis de ses instances nationales, sous l'égide du réalisateur Elem Klimov, lors du $\mathrm{V}^{\mathrm{e}}$ congrès de l'Union des cinéastes soviétiques, qu'il dirigeait alors. Indépendance confirmée en 1987, lors du XV ${ }^{e}$ Festival de Moscou, période faste qui vit les films censurés resurgir hors de leur enfer, mais vite brouillée par l'arrivée de l'économie de marché coïncidant, sur le plan cinématographique, avec l'oubli des grands cinéastes du passé et la mise en avant de nouveaux cinéastes s'adaptant aux goûts du public et du nationalisme. L'État reprenant rapidement sa place dans le contrôle du cinéma, déclarant qu'il accorderait "une bonne part de ses subventions aux projets montrant la Russie sous un jour positif ", et "véhiculant les valeurs patriotiques et morales ${ }^{7}$. Marilyne Fellous termine son texte par ce constat :

Est-ce à dire que la résistance, que le pouvoir autoritaire communiste n'était pas parvenu à écraser en 70 ans, a été vaincue par le pouvoir autoritaire capitaliste? Nous ne le pensons pas. Comme ils ont toujours su le faire dans les périodes les plus difficiles, les cinéastes russes commencent (lentement mais sûrement) à réagir aux pressions, et il faudra sans doute que le spectateur averti recommence à chercher dans les films russes les allusions assassines sur le régime en place. Mais verra-t-on bientôt un grand film sur les jeunes anti-fascistes d'aujourd'hui ou sur les nombreux journalistes assassinés, comme Anna Politkovskaïa, dont les assassins n'ont toujours pas été condamnés, nous ne le pensons pas8.

Entre le courage de Elen Klimov, ouvrant la possibilité d'une véritable réflexion sur l'histoire du cinéma soviétique et la lâcheté collaboratrice de Nikita Mikhalkov, entre les soubresauts d'autoritarisme et de nationalisme hérités des empires tsariste et communiste et les lois de l'économie de marché du cinéma commercial, se situe la grande complexité du positionnement des cinéastes les plus importants des dernières décennies. La présente réflexion portera sur certains d'entre eux, représentatifs du passage entre l'URSS et la Russie postsoviétique, qui se sont distingués par leur singularité, et sur des films portraits que leur ont consacrés des cinéastes occidentaux.

6. M. Fellous, «Le cinéma russe : vingt-cinq ans déjà », publié sur <http://www.kinoglaz. $\mathrm{fr} /$ marilyne_fellous_panorama_russe.php $>$.

7. Ibid.

8. Ibid. 


\section{Chris Marker : d'Alexandre Medvedkine à Andreï Tarkovski, ou du "ciné-train " révolutionnaire à la «métaphysique du travelling"}

Chris Marker a toujours entretenu avec le cinéma et la culture russes une relation très étroite, et mené une constante réflexion sur leurs accointances, ou leurs prises de distance avec le régime soviétique dans ses différentes phases. Pour lui, histoire du cinéma et Histoire entrent dans de multiples interactions, que son regard de réalisateur se doit d'interroger. Dans Lettre de Sibérie (1958), il dénonçait la propagande, et démontrait combien les mots d'un commentaire peuvent trahir le sens des images : des plans identiques y étaient par exemple accompagnés de trois textes off différents, l'un de propagande communiste, le second anticommuniste, le dernier presque neutre avec, dans chacun, une pointe d'ironie à peine voilée. Avant même d'en arriver à sa relation avec Andreï Tarkovski et son ouvre, exposée dans Une journée d'Andrë̈ Arsenevitch (montée en 1999 et diffusée en 200I, dans le cadre de la série "Cinéma de notre temps»), encore faut-il mentionner celle qu’il entretint longuement avec Alexandre Medvedkine, cinéaste ayant côtoyé les grands réalisateurs révolutionnaires que furent Eisenstein, Vertov, Koulechov ou Poudovkine. Cette rencontre se fit d'abord par l'intermédiaire d'un film, Le Bonheur (1934), qui devint le manifeste d'un petit groupe dont il faisait partie avec Jacques Ledoux, directeur de la Cinémathèque de Belgique (et interprète du rôle du savant fou de La Jetée 9 ). Le film de Medvedkine évoquait la révolution soviétique, mais contenait aussi des critiques sous-jacentes de certains de ses excès et, à d'autres moments, prenait une tournure surréaliste (le cheval à pois noirs, porté sur des épaules humaines dans un plan mémorable). Chris Marker admira aussi le "ciné-train", créé par Medvedkine pour sillonner l'URSS, qui permettait de filmer, développer, monter, projeter des films de manière autonome. Dans cette volonté de coller au réel, fut parfois saisi ce que le régime en place aurait souhaité masquer (Le Train en marche de Chris Marker, en 1971, relate cette aventure). Mais c'est dans Le Tombeau d'Alexandre (1993) ${ }^{\mathrm{I0}}$, consacré à la vie de Medvedkine, que Chris Marker donne à voir le saisissant parcours d'un homme et d'un cinéaste, et à travers lui celui de l'URSS, de ses prémices à son effondrement : de l'évidente nécessité de la révolution face à l'oppression du peuple par le pouvoir tsariste à l'après I99I,

9. Marker fonda le Groupe Medvedkine dans les années 1970, années militantes durant lesquellles Godard constituait parallèlement le Groupe Dziga Vertov.

Io. Voir D. Coureau, «Relations d'utopie : Chris Marker, Robert Kramer, Richard Dindo et les années 1990 ", CinémAction, $\mathrm{n}^{\circ} \mathrm{II} 5$, Utopie et cinéma, Y. Dureau (dir.), Corlet éd., 2005, p. 39-48. 
dans la déliquescence des anciennes institutions soviétiques, et la résurgence de courants de pensée réactionnaires. Ce film est peut-être le plus beau témoignage sur la fin de l'Empire soviétique. La voix off présente ainsi le cinéaste :

Alexandre Ivanovitch Medvedkine est un cinéaste russe né en 1900. Ces entailles que les pères de famille font aux chambranles des portes pour mesurer la croissance de leur progéniture, le siècle les a tracées sur sa vie. Il avait cinq ans, Lénine écrivait "Que faire?», dix-sept ans et il savait, vingt ans la guerre civile, trentesix ans les procès de Moscou, quarante et un ans la guerre, cinquante-trois ans Staline est mort, et quand il meurt lui-même en 1989, c'est dans l'euphorie de la perestroïka.

Le film de Chris Marker parcourt l'œuvre de Medvedkine, son utopie révolutionnaire et ses compromissions avec la propagande stalinienne, se partageant en deux parties symboliquement intitulées : «Le Royaume des ombres» (titre du texte que Maxime Gorki avait consacré à sa première expérience de spectateur du cinématographe Lumière), et «Les Ombres du royaume». Une archive montre Medvedkine, peu avant l'avènement de Mikhaïl Gorbatchev, déclarant : "Je vis depuis quatre-vingt-quatre ans et m'apprête à vivre encore le plus longtemps possible. Pas jusqu'au $\mathrm{XxI}^{\mathrm{e}}$ siècle... Cinq ans encore, ce serait bien...» La caméra montre alors la tombe du cinéaste, tandis que la voix off, véhiculant la pensée de Chris Marker, commente :

Et cinq ans plus tard tu es mort, le seul plan quinquennal qui ait jamais marché. Je suis retourné sur ta tombe le jour de Pâques, j'ai fait le tour des tombes amies" ${ }^{\mathrm{II}}$. Et quand titubant un peu dans la boue du dégel j'ai trouvé dans mon viseur un soldat tsariste $[\ldots]$ j'ai cru que j'avais forcé sur la vodka qu'on partage ce jour-là autour des tombes. Mais non, c'était bien le portrait d'Alexandre III et le tombeau symbolique des Romanov, et je n'avais encore rien vu [...]

Le film de Chris Marker s'attache alors aux aspects marquants de la période 1990-I99I. Du côté du dégel, ironiquement mentionné en relation avec la saison, le cinéaste évoque ainsi le moment où il accompagna Costa-Gavras pour la première diffusion de L'Aveu en juin 1990 à Moscou. Des plans actuels d'Yves Montand, dans la voiture qui les conduit au cinéma, alternent avec des plans de L'Aveu où Montand est transporté en voiture également, mais les yeux bandés. Ouverture symbolique du regard de la Russie sur un film concernant son histoire, tandis que la voix off souligne : "Nous pensions à ce que nous disions souvent pendant le tournage du film : "Le jour où l'on montrera ça en URSS, c'est que quelque chose aura vraiment changé" ». Si la résurrection

II. Parmi les tombes est montrée celle de V. Vyssotski, chanteur et acteur engagé qui fut le mari de M. Vlady et qui, auprès de L. Schwartzenberg et de Chr. Marker, fit partie du comité de soutien et d'accueil d'A. Tarkovski en France. 
du jour de Pâques coïncidait avec le retour symbolique du tsarisme, Marker évoque aussi, autre manifestation du dégel, une exposition sur la guerre en Afghanistan, étrangement objective, mais le commentaire précise aussitôt :

L'exposition ne durera que quelques jours, elle serait interrompue par le putsch, et ça serait la fin de l'utopie, pas la vôtre, l'idée même d'utopie, comme si l'Union soviétique avait été la dépositaire amnésique d'un espoir qu'elle aurait cessé depuis longtemps d'incarner mais qui étrangement mourrait avec elle. Encore quelques heures et c'en serait fini de la tyrannie et par la même occasion de la dissidence. Dans le paysage politique de l'après-putsch, les dissidents qui avaient été la conscience des années Brejnev ne joueraient plus aucun rôle, la porte était ouverte aux démagogues.

Ces derniers propos accompagnent les images du discours d'un orateur, Vladimir Jirinovski, transformant l'Histoire à renfort de nouveaux mensonges, tandis que la caméra scrute les visages de l'assemblée, dans l'espoir de saisir sur l'un d'eux un sursaut de conscience dissidente. Au cour d'Une journée d'Andreï Arsenevitch, Medvedkine fait une réapparition en 1988, un an avant sa mort, déclarant à Chris Marker :

Je suis très affligé par la disparition d'Andreï Tarkovski. Nous sommes tous très affligés. Il était le préféré de notre collectif de cinéma. Je sais la peine qui est la tienne. Je dois te dire que notre collectif n'approuve pas la condamnation qui a été faite de ses actes. Nous avons ressenti la perte de cet homme formidable, intéressant, avec la même douleur que toi et que beaucoup de gens à Paris. Aujourd'hui, tout cela ne pourrait plus arriver...

Et la voix off du film, celle de Marina Vlady transmettant la pensée de Marker, poursuit :

Oui, mais c'était arrivé, et l'hommage d'un vieux bolchévique n'efface pas vingt ans de tracasseries en tout genre. [...] Un harassement continuel qui le conduira à choisir l'exil, lui qui était si naturellement et profondément russe.

Chris Marker souligne que Tarkovski, «étranger en ce monde», refusant catégoriquement de se considérer comme un dissident, était proche de la figure mythique du yurodivi (l'innocent) de l'opéra de Moussorgski (d'après Pouchkine) Boris Godounov, dont Tarkovski fit une mise en scène. La fin de l'existence de Tarkovski, point de départ du film de Chris Marker, situe le cinéaste russe au bord de cette ligne de fissure qui conduisit, cinq ans plus tard, à l'effondrement de l'URSS. Tarkovski a commencé à travailler dans les années I960 - non loin du Printemps de Prague; sa mort intervient après l'arrivée au pouvoir de Mikhaïl Gorbatchev, alors qu'il a choisi l'exil, pour demeurer définitivement en Occident en 1984, juste après avoir réalisé Nostalghia (où il disait avoir exprimé la nostalgie russe ressentie dans l'éloignement de la terre natale) en Italie en 1983. Dans Une journée d'Andrë̈ Arsenevitch, Chris Marker entremêle plusieurs types d'images : des plans de Tarkovski filmés en 1986, peu 
après qu'il eut appris, en plein montage du Sacrifice - titre éloquent, pour un film qui s'avérera testamentaire - qu'il était atteint d'un cancer ${ }^{\mathrm{r} 2}$; d'autres plans de Tarkovski au travail, lors du tournage du Sacrifice en Suède; des extraits de ses films, mis en relation par le montage de Marker pour éclairer l'existence et la pensée du cinéaste (rapport au pouvoir et lutte contre la censure, rapport au monde, aux éléments naturels, à la culture et à la mystique russes, attachement contrarié à la terre et à la maison familiales). Tous ces niveaux de perception sont traversés par la voix off qui semble, sans cesse, regarder les images et en dévoiler le sens caché. La captation du tournage d'un plan séquence complexe en Suède, par Chris Marker, peut traduire à elle seule l'art de Tarkovski. Dans ce plan de six minutes, dit le commentaire, «l'œil suit le personnage qui vient de mettre le feu à sa maison, sa famille qui le rejoint et s'affole, l'ambulance qui arrive, tout cela dans le même rapport au cadre, et porté uniquement par le va-et-vient de la caméra». La première prise ayant échoué, Tarkovski s'était retrouvé dans la nécessité de réussir la seconde et dernière prise possible. Voici ce que Chris Marker, via la voix off, dit de ce dispositif :

L'exigence de ce plan unique n'est pas un caprice de metteur en scène virtuose. Ici les quatre éléments jouent ensemble. La terre et l'eau, étroitement mêlées, la mer libératrice à l'horizon, le feu apporté par l'homme, et l'air qui enveloppe tout, signifié par cet espace, cet éloignement des bords qu'en langage de photographe on appelle justement "de l'air». Traiter cela de manière classique, plans rapprochés, contrechamps, inserts de l'incendie, aurait tout faussé. L'action doit tenir dans un seul cadre, où le travelling n'est plus affaire de morale, mais de métaphysique.

Cette idée de «métaphysique du travelling" s’inscrit dans la lignée d'une réflexion entamée de longue date par les Cahiers du cinéma, ayant conduit à penser une "morale du travelling", interrelation étroite entre forme et sens (Luc Moullet, Jean-Luc Godard, Jacques Rivette, Serge Daney). Cette alliance des termes montre à quel point, pour Chris Marker, la pensée de Tarkovski se situe dans une sphère spirituelle, et combien cette pensée complexe ne pouvait s'accorder à la pensée matérialiste dominante du régime socialiste soviétique. Chris Marker, en un autre moment, donne à voir les derniers plans du film de Tarkovski, Andrë Roublev, consacrés aux icônes du peintre et dans lesquels, remarque-t-il, figurait déjà la peinture abstraite de Malevitch. Par là, il signifie que le cinéma de Tarkovski entrecroisait pensée classique et plus extrême modernité du xxe siècle. Chris Marker suit aussi un

I2. Les plans les plus émouvants sont ceux de l'arrivée d'Andrioucha, «libéré» in extremis par les autorités russes afin de rejoindre ses parents, Andreï et Larissa Tarkovski. De ces plans filmés par Marker, Tarkovski écrit dans son Journal : "Je suis très reconnaissant à Chris d'avoir filmé pour nous cet instant - peut-être le plus important de ma vie» (A. Tarkovski, Journal (1970-1986), Cahiers du cinéma, I993, p. 400). 
autre fil, disant de Tarkovski qu'il est «le seul cinéaste dont l'œuvre entière tient entre deux enfants, et entre deux arbres", ce qu'il illustre par le premier plan de L'Enfance d'Ivan et le dernier plan du Sacrifice, celui de l'enfant qui a juste recouvré la parole, allongé au pied de l'arbre sec qu'il tente de faire revivre en l'arrosant chaque jour, bientôt quitté par un travelling qui s'élève vers la cime de l'arbre et le ciel. Le Sacrifice est d'ailleurs dédié par Tarkovski à son fils, et il est évident que la symbolique de l'arbre renaissant associée à celle de l'enfance est un signe d'espérance par-delà la lutte de l'artiste avec le pouvoir. Lors de l'examen du film Solaris par les instances officielles, parmi une invraisemblable liste de remarques, figuraient les suivantes : "Supprimer le concept de Dieu», "Supprimer le concept de christianisme», "On ne comprend pas que l'océan soit doué d'intelligence $\aleph^{13}$. Dans le dernier entretien accordé avant sa mort, Tarkovski déclarait : «L'espoir a plutôt tendance à se renforcer face au plus sordide de notre société actuelle. Tout simplement parce que l'horreur, tout comme le beau, provoque des sentiments qui, chez un croyant, renforcent l'espoir ${ }^{\mathrm{I4}}$.» L'opposition au régime de l'URSS, par la création et la mise en avant du créateur (artiste - peintre, cinéaste -, penseur, mystique), s'était aussi doublée d'une nécessaire lutte contre le tournant économique que Tarkovski avait eu le temps de pressentir dans les années 1980 : "Le cinéma est tombé dans la nullité. Avant tout parce qu'il s'est complètement coupé du monde intérieur de ceux qu'on appelle les cinéastes. Pour eux, le cinéma n'est plus qu'un gagne-pain facile et une façon de recevoir des honneurs. Je veux faire un film qu'on puisse comparer, de par sa profondeur, à un acte de vie», écrivait-il dans son Journal' ${ }^{15}$. Le film de Chris Marker se clôt par l'évocation des funérailles de Tarkovski qui eurent lieu à la cathédrale Nevsky de Paris, une cathédrale qui a non seulement «un nom de film» comme le souligne le commentaire, mais qui associe l'idée religieuse à la mémoire du cinéma. Ailleurs dans le film, Chris Marker avait relaté, et illustré, la prophétie que Tarkovski avait reçue de Boris Pasternak lors d'une séance de spiritisme, selon laquelle il ferait sept films. C'est dans la capitale française que Tarkovski termina son existence, à la suite de la réalisation des sept films que comporte effectivement sa filmographie. Sa résistance se situe en dehors de la dissidence politique et si, dans ses films, l'enfermement, l'oppression, la menace constante de la violence et de la guerre figurent sans cesse - de L'Enfance d'Ivan au Sacrifice où l'idée d'une guerre nucléaire est

I3. Ibid., p. 59.

I4. A. Tarkovski, Th. Jonhson, "Une lueur au fond du puits?», entretien du 28 avril 1986, <publié sur http://www.cles.com/entretiens/article/une-lueur-au-fond-du-puits >.

I5. A. Tarkovski, Journal (1970-1986), ouvr. cité, p. 73. 
suggérée, en passant par Stalker et sa "Zone ${ }^{16}$ ", ou par la science-fiction de Solaris-, ce qui est toujours en jeu dans son œuvre c'est la lutte d'un homme seul contre la société, qui se joue avec des armes métaphoriques - geste hautement politique, au sens philosophique du terme.

\section{Anne Imbert : Alexandre Sokourov ou l'élégie de l'Histoire}

Dans l'un des premiers articles consacrés à Alexandre Sokourov par la presse française, publié dans les Cahiers du cinéma en 1987, les propos suivants du cinéaste sont cités : "Puisque nous sommes vivants, ou plutôt puisque nous avons survécu, nous pouvons encore faire beaucoup. Les réserves émotionnelles de la Russie sont inépuisables ${ }^{17}$." Le constat d'un dépassement de l'URSS est déjà décelable ici, associé à un profond enracinement dans une idée de Russie éternelle, en ce moment de tournant de la perestroïka et de la glasnost. Une vingtaine d'années plus tard, c'est un cinéaste, à présent connu et reconnu en Europe occidentale, qui apparaît dans le film que lui consacre Anne Imbert - film peu diffusé, produit par la chaîne de télévision France 3 Alsace - intitulé Alexandre Sokourov: questions de cinéma (2008). Ici, la forte subjectivité de Chris Marker, sa présence constante dans le montage et dans le texte, son approche essayiste de Tarkovski, sont remplacées par une sobriété esthétique qui, néanmoins, trace un portrait éclairant d'Alexandre Sokourov. Anne Imbert a choisi de rencontrer le cinéaste dans sa ville, Saint-Pétersbourg. De ces lieux, sont captés des extérieurs : au début du film, des variations sur les nuages sont proposées, dans la proximité des «élégies» filmées par Alexandre Sokourov, puis interviennent au cours du film des plans de ruelles, de quais le long de la Neva, de zone portuaire, du musée de l'Ermitage (que le cinéaste considère comme le lieu central de la ville, principal motif de sa présence dans celle-ci, la peinture occupant la plus haute place dans sa vision de l'art). Alexandre Sokourov est suivi dans ces différents lieux, poursuivant le fil de la conversation qui est aussi filmée, en plusieurs autres moments, en plans rapprochés, dans des intérieurs. Les images des extérieurs constituent des passages qui, en s'approchant de l'esthétique même d'Alexandre Sokourov, permettent d'accéder aux extraits de ses films. À ces séries (plans dans la ville, extraits), s'en ajoutent deux autres,

16. Dans son Journal, Tarkovski relate qu'A. Vitez avait dit que Stalker est un film «qui l'a obligé à réfléchir, pour la première fois, sur la fin du monde et sur la fin du socialisme» (ibid., p. 306).

17. A. Sokourov, cité dans Fr. Albera, "URSS : voyage dans un cinéma en mutation ", Cahiers du cinéma, $\mathrm{n}^{\circ}$ 395-396, Ciné-Monde la nouvelle ligne, mai 1987, p. I03-I04. 
scripturales, qui viennent structurer le film. La première est composée de cartons - blancs ou noirs - sur lesquels sont inscrits des titres de chapitres, repris des propos d'Alexandre Sokourov : "Courbes", "La mélodie met tout en place», "Torsions et distorsions», «Désaccord et accord», «Existe-t-il un silence de l'image?", "Les poussières du chemin». La seconde consiste en une énumération de titres de films qui viennent progressivement s'inscrire en blanc sur fond noir, selon trois thématiques : «Parenté» qui réunit les films Le Jour de l'éclipse, Le Deuxième Cercle, Mère et fils, Père et fils, Alexandra, où apparaît la figure grand-maternelle (films réalisés entre 1990 et 2003); «Élégies»: Élégie paysanne, Élégie, Élégie de Moscou (consacrée à Tarkovski, et incluant certaines images de Chris Marker), Élégie soviétique, Élégie pétersbourgeoise, Élégie simple, Élégie de Russie, Élégie orientale, Élégie de la traversée $e^{18}$ (films de 1978 à 2006); "Histoire»: Moloch (sur Hitler), Taurus (Lénine), Le Soleil (Hiro Hito), L'Arche russe (films réalisés de 1999 à 2002) ${ }^{19}$.

Quelques phrases extraites d'entretiens au sujet du film d'Anne Imbert permettent de traverser toutes les séries définies ci-dessus. Sur la famille, Alexandre Sokourov affirme qu'en Russie "la distance entre père et fils est colossale. [...] Le père, d'une manière générale, est une figure de peu d'importance, temporaire, qui vient de temps en temps. Tout le monde est concentré sur la mère [...]", précisant que le père n'est reconnu qu' "une fois qu'il a disparu ». Dans ce contexte, Alexandre Sokourov, fils de militaire, pense que l'homme, en raison de «ce monde féminin qui l'entoure», trouve un milieu qui le comprend au sein de l'armée. Milieu qu'il décrit de manière très paradoxale, puisqu'il le définit par «une compréhension absolue des uns et des autres, des intérêts et des goûts communs», et pense qu'il rassemble les hommes «les plus faibles [...], les plus fragiles», car quelqu'un qui sait qu'il peut mourir « fait tout son possible pour survivre et [...] mettre en avant ses côtés les plus faibles». Le film choisi par Anne Imbert pour illustrer ces propos, consacré à l'armée, porte du reste ce titre décalé : Voix spirituelles. Cette étrange conception explique très certainement le traitement non réaliste de la guerre par Alexandre Sokourov, et l'extension de cette quasi-mystique de l'armée fraternelle à ses films tournés en Tchétchénie, dont Alexandra est l'emblème. À Jean-Michel Frodon qui s'étonnait de l'absence du mot «Tchétchène» dans celui-ci, Alexandre Sokourov rétorqua : «Pour moi la

18. Voir D. Coureau, "Élégie de la traversée : les voix spirituelles du temps", CinémAction, $\mathrm{n}^{\mathrm{o}}$ 133, Alexandre Sokourov, Fr. Albéra, M. Estève (dir.), Corlet éd., 2009, p. I26-133.

19. Il est à noter que, depuis, A. Sokourov a signalé que les trois premiers de ces films constitueraient une tétralogie avec son Faust, inspiré de Goethe et du Docteur Faustus de Thomas Mann (ouvrage que Tarkovski avait pensé adapter en 1973). 
Tchétchénie fait partie de mon pays ${ }^{20} »$. Du reste, les responsables des pires crimes contre l'humanité, Hitler, Lénine (qu’il soit inclus dans ce triptyque en dit long sur l'opposition frontale à l'Histoire officielle soviétique), Hiro Hito ne sont pas non plus traités en figures dominatrices, mais au moment de leur déchéance, politique, physique et mentale, dans la médiocrité du quotidien le plus banal. Le rapport à l'Histoire qu'entretient Alexandre Sokourov est avant tout un rapport de deuil. Parmi les plus beaux plans du film d'Anne Imbert figurent ceux du musée du Blocus de Léningrad et du cimetière Pisskarev des victimes de ce Blocus. Les tombes collectives, immenses, géométriques, couvertes de pelouse, et séparées par de longues allées sont données à voir, ainsi que des portraits sortant les morts de leur anonymat, accrochés sur les troncs des arbres. Alexandre Sokourov commente : "Il n'y a pas un seul endroit vivant dans cette ville. La mort est partout et on a payé un tel prix, je suis certain qu'une ville ne mérite pas tant de vies humaines.» Face à quelques objets filmés dans le musée, pauvres ustensiles (tasse en fer, assiette, réchaud...), il s'interroge :

Pourquoi donc les Russes, accablés de cette lourdeur depuis tant de siècles, ont pu résister aux nazis $[\ldots]$ ? C'est une question difficile [...]. Deux millions de civils ont péri ici, à Léningrad, pendant le Blocus, et des centaines de milliers de militaires avec eux. Imaginez ce qu'il y avait à l'intérieur de ces gens. Imaginez cette complexité contradictoire, que même le bolchevisme n’a pas su détruire.

Cette prégnance de la mort qu'Alexandre Sokourov révèle dans son approche de la famille, de l'armée, des figures les plus négatives de l'Histoire mondiale $\mathrm{du} \mathrm{XX}^{\mathrm{e}}$ siècle, de l'histoire de la ville qu'il a décidé d'habiter, détermine aussi le choix qu'il a fait d'une esthétique entièrement fondée sur le sentiment élégiaque, mélange de tristesse et d'impuissance face au cours des choses, mélancolie persistante qui guide ses choix visuels d'une image souvent décolorée, floue ou voilée, dans des climats brouillés : nuages, neige, pluie, brumes, brouillards, eaux du fleuve ou de la mer, parfois saisis dans des surimpressions, des jeux de déformation optique, et ses choix sonores : musiques classiques profondes, parfois pesantes (Tchaïkovski, Mahler, Glinka...). Un unique flux de pensée et de sentiment semble composer l'esthétique de ses films, lente et longue méditation sur la mort, individuelle aussi bien qu'historique. Le glissement du théâtre Nô (du côté de son affinité avec la pensée extrême-orientale) aux peintures de ruines nocturnes d'Hubert Robert, puis au musée de l'Ermitage la nuit et aux eaux de la Neva, dans un court métrage dédié au peintre du XviII ${ }^{\mathrm{e}}$ siècle, semble définir la traversée onirique qu'Alexandre Sokourov fait de l'Histoire et de l'histoire de l'art tout aussi bien. Dans le film d'Anne Imbert, Alexandre Sokourov 
définit l'élégie comme "une réflexion ou une réminiscence de quelque chose qui est passé, qu'on aimerait faire revenir mais qui ne reviendra jamais. [...] Pourquoi? Parce qu'il y a la mort», puis il précise :

Non, on ne peut pas dire que le sentiment élégiaque ou poétique, avec toutes ses nuances, porte une prédestination. Bien sûr, la tristesse est une qualité divine [...]. L'être humain est-il le seul à être doué de cette qualité, ou d'autres êtres l'ont-ils aussi? [...] Pourquoi les gens capables de vivre dans cet état élégiaque de tristesse, pourquoi sont-ils capables de supporter des situations extrêmement difficiles de leur vie, des fardeaux extrêmes, tandis que ceux qui ont un caractère léger $[\ldots]$ ne peuvent pas porter de tels fardeaux?

Radicalement, Alexandre Sokourov dit aussi attendre l'apparition d'«un nouvel art sur les ruines du cinéma qui s'est discrédité et qui fait d'immenses dégâts aux sociétés humaines. [Car] aujourd'hui, le cinéma est l'ennemi principal de l'humanité». Mais il en appelle aussi à développer un "côté humaniste du cinéma", même si celui-ci est menacé par la télévision et les financiers. Sa conclusion, à la fin du film d'Anne Imbert, est une recommandation adressée à un hypothétique cinéaste à venir : «toi-même, toi-même, fais tout toi-même $[\ldots]$. Ne compte sur personne, ne compte même pas sur ton père et ta mère, et ne compte sûrement pas sur Dieu. Fais tout toi-même». Le dernier plan du film montre Alexandre Sokourov, de dos (figure souvent présente dans son cinéma), s'éloignant d'une démarche un peu lourde, dans un couloir, illustration de cet état de solitude nécessaire à la création.

\section{Julie Bertucelli : Otar Iosseliani ou la fable de l'Histoire}

Dans le dossier de presse de son film de 20Io, Otar Iosseliani explicite la signification du titre qu'il lui a choisi, Chantrapas:

C'est du russe, inspiré du français "chantera pas». À la fin du XIXe siècle, toutes les familles aisées de Saint-Pétersbourg amenaient leurs enfants à des maîtres de bel canto italiens pour leur apprendre le chant. À l'époque, l'aristocratie russe parlait français, donc les Italiens avaient appris deux mots, lorsqu'ils sélectionnaient les enfants : «chantera» et «chantera pas». Ensuite, chantrapas est devenu un nom commun : les "chantrapas" étaient des bons à rien, les exclus... Un peu comme mon personnage principal censuré en Union soviétique, et moins bien reçu en Occident qu'il ne l'espérait. Victor Hugo, Fritz Lang [...] Tarkovski [...]... tous étaient des exclus, des "chantrapas», obligés de quitter leur pays natal, sans bien savoir nager dans ces eaux inconnues, tous portant une blessure.

Le terme chantrapas s'accorde dès lors tout à fait à Iosseliani lui-même, qui s'est expatrié au début des années I980 afin de pouvoir poursuivre son œuvre, à Paris, ville qu'il trouva décevante par rapport à la mythologie qu'il s'en était forgée (de Dumas à Queneau, de Carné à Tati...), trouvant à la place de la 
vie culturelle attendue une muséification des souvenirs. Otar Iosseliani est né à Tbilissi, capitale de la Géorgie, et sa position diffère de celle d'Andreï Tarkovski et d'Alexandre Sokourov, tous les deux russes. Otar Iosseliani s'est attaché à l'Histoire de son pays, auquel il a consacré un documentaire en trois parties, Seule Géorgie (pour une soirée thématique d'ARTE en 1994) : «Prélude» (des origines au XvıII ${ }^{\mathrm{e}}$ siècle), «Tentation» (de la révolution soviétique à la perestroïka), "L'épreuve» (sur les bouleversements de l'après-chute de l'empire, et la guerre civile qu'elle déclencha en Géorgie). Il était, qui plus est, fils d'un officier de l'armée du tsar, qui connut le goulag, ce qui le différencie encore d'Andreï Tarkovski, fils d'un poète russe célèbre, et d'Alexandre Sokourov.

Julie Bertucelli a pu affirmer son attachement à la culture russe, et son intérêt particulier pour la Géorgie, carrefour entre Europe et Asie (elle a d'ailleurs été assistante d'Otar Iosseliani). Cet attrait s'est confirmé lors de son passage du documentaire à la fiction, dans Depuis qu'Otar est parti (2004) ${ }^{21}$, film qui commence à Tbilissi et se termine à Paris, suivant un cheminement qu'Otar Iosseliani connaît bien. Le film de Julie Bertucelli consacré au cinéaste s'intitule Otar Iosseliani, le merle siffleur (2006, dans la série "Cinéma de notre temps»), et suit des moments de l'écriture, du tournage et de la sortie d'un film du cinéaste géorgien, comme un carton le précise : «2005-2006, Otar Iosseliani réalise Jardins en automne» (qui s'intéresse à un ministre déchu retrouvant le goût de la musique et du jardinage, fable en fait sur le pouvoir qui permet à Otar Iosseliani de préciser sa philosophie dans le dossier de presse : «Depuis les fables d'Esope, bien copiées par La Fontaine en France, on sait combien la parabole a toujours une base dans la réalité»). Si Julie Bertucelli, dans une grande proximité avec le cinéaste, donne la primauté au filmage de son travail en train de se faire, elle intègre aussi à son montage des extraits de films (certains étant cités à plusieurs reprises): Il était une fois un merle chanteur (197I, dont le personnage de musicien, en marge des institutions, est très représentatif du positionnement d'Otar Iosseliani) et La Chute des feuilles (1967) du côté des films géorgiens; La Chasse aux papillons (1992, fable où les héritiers décadents de la noblesse géorgienne se heurtent à Paris aux réalités économiques du capitalisme), Lundi matin (2002, où Otar Iosseliani campe un faux grand pianiste, jouant en play-back sur de la musique enregistrée), Brigands chapitre VII (I996, illustration décalée d'une éternelle guerre civile géorgienne, où les chars s'insèrent dans la vie quotidienne), Adieu plancher des vaches (I999, où Otar Iosseliani apparaît aux côtés de son alter ego, échassier de l'espèce des

2I. Voir D. Coureau, "Trois femmes en clair-obscur : Depuis qu'Otar est parti», Contre Bande, $\mathrm{n}^{\circ} \mathrm{I} 8$, "Le cinéma féminin français contemporain", université Paris I - PanthéonSorbonne, 2008, p. 93-I06. 
marabouts). À la fin du film, vient aussi s'afficher, sur fond noir, l'intégralité des titres des films d'Otar Iosseliani. Comme Anne Imbert s'accordait à la mélancolie de Sokourov dans son filmage, Julie Bertucelli trouve dans le sien le ton distancié de la fable, pour filmer Otar Iosseliani jusque dans ses moments de doute et d'attente, lorsqu'il fume ou réclame du vin pour continuer à créer. Dans les premiers plans du film, comme un prégénérique, Iosseliani marche dans les rues nocturnes de Cannes où son film va être présenté lors du Festival. Il se met à citer des vers de Pouchkine qu'il dit intraduisibles. Plus tard, il est saisi au travail face à une infinité de papiers épinglés aux murs, où figurent des fragments de phrases-séquences, vaste cadavre exquis à ordonner dans le film à venir. Face à l'un de ces papiers, "Le contraire du merle chanteur», Julie Bertucelli s'interroge, et Otar Iosseliani précise : "Ah, le contraire du merle chanteur, c'est un type qui travaille tout le temps.» Lors du tournage d'un plan en appartement, Michel Piccoli - qui interprète le personnage d'une vieille femme! - évoque avec le cinéaste les photographies qui sont au mur : photographies de la propre famille du cinéaste, témoignages d'un monde oublié, d'une vieille noblesse géorgienne, dont ne subsistent que quelques traces. Employant le terme de dissident pour se caractériser, Otar Iosseliani aime ajouter qu'il s'agit surtout d'un «terme fourre-tout qui avait valeur de vademecum en Occident ${ }^{22} »$. Il est facile de comprendre que le cinéaste, par ses origines familiales, et son attitude peu encline à suivre les directives de quelque autorité que ce soit, s'il avoue avoir "eu une évolution très lente" sur le plan politique, «d'autant plus qu'en Géorgie, il n'y avait pas d'hystérie idéologique, mais toujours de l'ironie ${ }^{23} »$, s'est trouvé en constante opposition avec le pouvoir et avec la censure. Ne serait-ce que dans la lutte entre un monde disparu, consciemment idéalisé, régi par la culture et par l'art (la musique et le chant sont centraux), et les régimes politiques successifs. Otar Iosseliani résume ainsi l'Histoire récente de la Géorgie :

Dès que la Géorgie fut indépendante, les malheurs ont commencé. Si la Géorgie veut ressembler à la Russie actuelle, c'est la catastrophe. C'est déjà la mafia qui dirige le pays avec une prime encouragée par le mauvais goût. Je crains fort que [...] l'enrichissement à tout prix, ne parvienne à un résultat auquel ni la colonisation tsariste ni l'occupation soviétique n'étaient parvenues : rendre les gens sauvages et incultes ${ }^{24}$.

22. O. Iosseliani, cité par G. Lefort dans «Iosseliani l'URSS mal léché», publié sur <http://next.liberation.fr/cinema/oror2291559-iosseliani-l-urss-mal-leche> [mis en ligne le 22/09/20I0].

23. O. Iosseliani, S. Kaganski, «Scepticisme d'Otar : Otar Iosseliani», entretien publié sur <http://www.lesinrocks.com/actualite/actu-article/t/I2234/date/I997-OI-22/article/ le-scepticisme-dotar-otar-iosseliani/ $>$.

24. O. Iosseliani, cité dans G. Lefort, art. cité. 
Le heurt avec les puissances de l'argent se trouve métaphorisé dans le film de Julie Bertucelli lors des confrontations entre le cinéaste et sa productrice, Martine Marignac, intermédiaire entre les financiers et le cinéaste (un partenaire russe fera symboliquement faux bond en cours de réalisation), qui représente aux yeux d'Otar Iosseliani une forme d'autorité qui ne peut comprendre les véritables nécessités créatrices : résurgence des luttes politiques contre la censure soviétique, à présent remplacées par des luttes économiques. Otar Iosseliani tente ainsi de sauvegarder ce qu' on appelle «en musique», dit-il, le " rubato ", le rythme de son film. Emblématique est cette remarque de Martine Marignac : "On a suffisamment de personnages comme ça. Savoir qu'il est insomniaque, qu'il lit Rabelais, on s'en fout comme de sa première chemise." Otar Iosseliani se défend : "Chaque mot que tu prononces, ça détériore la situation. Moi je suis fragile, ça finit par me donner des doutes», et Martine Marignac conclut : "C'est tout à fait le but de la manœuvre!» Un dialogue surréaliste les oppose sur la nécessité de tourner des plans où des sangliers courent dans la nature, à trois-cent-cinquante kilomètres de Paris : "filmer cette saloperie de cochon coûte cinq mille euros, est-ce raisonnable dans l'économie où nous sommes?» argumente Martine Marignac! C'est néanmoins par ces plans, au moment de leur tournage, en lisière d'une forêt sous la pluie, que Julie Bertucelli montre que l'obstination créatrice d'Otar Iosseliani a fini, cette fois-ci, par l'emporter sur les raisons économiques. Et c'est à la fin de cette séquence que le cinéaste déclare, en guise de conclusion de son tournage et, simultanément, du film de Julie Bertucelli : «On va continuer de vivre maintenant.»

\section{D'un Empire à l'autre...}

Dans le dossier de presse de Chantrapas, Otar Iosseliani déclarait : "C'est une parabole sur la nécessité de rester soi-même malgré tous les obstacles qui nous entourent", rejoignant les propos de Sokourov sur la nécessité d'être soi-même. Vision guidée par la place de l'artiste dans le monde pour Andrë̈ Tarkovski, vision élégiaque d'Alexandre Sokourov, vision ironique-satirique d'Otar Iosseliani se rejoignent dans l'affirmation d'une indispensable singularité créatrice, et d'une opposition au contrôle politique et financier : «Il n'y a que ce qui filtre à travers le prisme de la vision subjective de l'auteur qui devient le matériau de création et qui peut former un monde complexe unique, un reflet de la vie réelle», écrivait Andreï Tarkovski ${ }^{25}$. Dans des propos

25. A. Tarkovski, Le Temps scellé, Cahiers du cinéma, I989, p. 32. 
proches de ceux d'Otar Iosseliani, Alexandre Sokourov - qui a aujourd'hui pris ses distances avec le - en vient à déclarer : «À voir la tournure que prend ma vie aujourd'hui, je peux dire qu'il vaut mieux travailler avec un régime totalitaire, disons plutôt lutter contre celui-ci, contre la censure qu'il exerce à l'égard des artistes, qu'avec les gens et les problèmes actuels en Russie, la pression des investisseurs privés ${ }^{26}$ ", ajoutant de manière paradoxale : "C'est en Union soviétique que se trouvait la meilleure conjoncture économique pour le cinéma, depuis les années i920 jusqu'au départ de Gorbatchev ${ }^{27}$.» Mais Iosseliani va aussi très loin, dans ses propos sur la Géorgie et sa renaissance douloureuse à l'indépendance :

J'ai pu observer que les mêmes gens qui étaient dans la nomenklatura ont changé de cap : ils sont devenus des mafieux, alias des gens d'affaire, ou sont entrés dans la mouvance libératrice nationaliste. [...] Ainsi, après la chute du communisme, alors que nous pensions que tout allait changer, rien n’a changé dans l'essentiel. Il y a eu un déplacement de quelques éléments de société, mais, aux plus hauts échelons du pouvoir, sont réapparus les mêmes personnages, sous un masque différent $\mathrm{t}^{28}$.

La chute de l'Empire soviétique n'a pas coïncidé avec une libération totale de l'art, ni dans la Russie d'Alexandre Sokourov (où capitalisme et autoritarisme se conjuguent), ni dans la Géorgie d'Otar Iosseliani (en proie au nationalisme et à l'instabilité politique et territoriale), et ces cinéastes autrefois censurés n'ont de cesse de continuer leur œuvre, sous forme d'élégie ou de fable et, ici à Saint-Pétersbourg, ou ailleurs dans l'exil, d'affirmer la différence irréductible de leur pensée à travers leurs films.

26. A. Sokourov, A. Cattin "Ostrov Sokourova : l'île Sokourov», entretien publié sur $<$ http://www.hors-champ.ch/HC_I/HC_I_OSTROV.PDF>.

27. Ibid.

28. O. Iosseliani, M. Marignac, P.-A. Boutang, entretien publié sur <http://yrol.free.fr/ CINEMA/IOSSELIANI/iosseliani.htm>. 